\title{
SEXISME ET FÉMINISATION DE LA LANGUE: UN PROCESSUS DE MANUÉLISATION*
}

\author{
Dantielli Assumpção Garcia ** \\ Universidade Estadual do Oeste do Paraná \\ Centro de Comunicação, Educação e Artes \\ Cascavel, PR, Brasil
}

\begin{abstract}
Résumé : À partir de l'articulation théorique entre l'Analyse de Discours et l'Histoire des Idées Linguistiques, ce travail analyse le manuel Femme, j'écris ton nom... Guide d'aide à la féminisation des noms de métiers, titres, grades et fonctions (1999), dans le but de réfléchir sur la constitution du processus de manuélisation d'un langage non sexiste marqué par la féminisation de la langue. La manuélisation d'une langue féminisée produit un savoir sur le genre morphologique, ce qui permet d'expliciter la formation de la forme féminine des noms de métiers, fonctions, grades et titres, et le manuel Femme, j'écris ton nom ... met en évidence que les femmes peuvent exercer (ou exercent déjà) n'importe quel métier et que l'emploi du masculin comme forme générique est une erreur et une agression contre la femme.
\end{abstract}

Mots-clés : Féminisation. Manuélisation. Analyse de Discours. Histoire des Idées Linguistiques.

1 INTRODUCTION

Dans ce travail, à partir de la perspective théorique de l'Analyse de Discours et de l'Histoire des Idées Linguistiques, on utilise les notions d'outil linguistique, grammatisation (AUROUX, 1992) et manuélisation (PUECH, 1998) afin d'analyser le manuel Femme, j'écris ton nom... Guide d'aide à la féminisation des noms de métiers, titres, grades et fonctions, publié en France, en 1999. Ce manuel constitue un savoir sur un «langage non sexiste », c'est-à-dire la matérialisation du genre féminin dans le fonctionnement linguistique. On cherche à comprendre la manière dont ce savoir circule dans la société et formule un dire qui matérialise la présence de la femme dans la langue. On cherche donc à répondre à ces questions : 1. Quel est le genre (morphologique ? socioculturel ?) présent dans ce manuel ?2. Comment un savoir linguistique qui porte sur la féminisation de la langue est-il disciplinarisé ? 3. Qu'est-ce que féminiser la langue ? 4. Comment peut-on comprendre ce processus de manuélisation d'un langage non sexiste?

\footnotetext{
* Ce travail fait partie de ma recherche postdoctorale intitulée A manualização do saber linguístico e a constituição de uma linguagem não sexista [La manuélisation du savoir linguistique et la constitution d'un langage non sexiste], à l'Université Estadual do Oeste do Paraná, sous la supervision du Prof. Dr. Alexandre Sebastião Ferreira Soares (PNPD-CAPES).

** Doutora em Estudos Linguísticos pela Universidade Estadual Paulista (UNESP). Docente dos Cursos de Letras e da Pós-Graduação em Letras da Universidade Estadual do Oeste do Paraná (UNIOESTE). Email: dantielligarcia@gmail.com.
} 
Notre but est de montrer que la construction d'un langage non sexiste de genre à partir de la féminisation de la langue est une proposition présente dans différents pays, qui essaient de répondre aux revendications féministes pour une position légitimée à la femme, non seulement dans la société, mais aussi dans les usages de la langue dans cette société-là. Basé sur l'Analyse de Discours et l'Histoire des Idées Linguistiques, notre travail incite donc des discussions pertinentes sur les notions de manuélisation et grammatisation d'une langue. Dans le cadre des théories féministes, ce travail nous aide à réfléchir sur l'importance des études qui établissent un rapport entre le langage et les questions de genre. Finalement, en provoquant une discussion théorique sur l'analyse d'outils linguistiques, ce travail provoque aussi une réflexion sur les conditions actuelles de production d'un dire sur le langage (non) sexiste.

Notre texte est divisé en quatre parties. Tout d'abord, nous présentons notre perspective théorique et la manière dont nous comprenons le processus de manuélisation et de grammatisation d'une langue. Ensuite, nous parlons du sexisme dans le langage et nous analysons ses formes de manifestation. Après cela, nous traçons un panorama théorique sur la féminisation d'une langue. Finalement, nous analysons le manuel Femme, j'écris ton nom... Guide d'aide à la féminisation des noms de métiers, titres, grades et fonctions (Centre National de la Recherche Scientifique, Institut National de la Langue Française, 1999), afin de comprendre ses propositions de féminisation et de constitution d'un langage non sexiste.

\section{LE PROCESSUS DE MANUELISATION}

Dans ce travail, nous nous inscrivons dans l'articulation entre l'Analyse de Discours et l'Histoire des Idées Linguistiques (HIL). D'après Eni P. Orlandi (2001), l'approche discursive «nous permet d'analyser les discours du et sur le langage, ce qui nous aide à obtenir nos résultats et [...] nous permet de pratiquer de nouvelles lectures d'archive »(ORLANDI, 2001, p. 16, nous traduisons). En outre, l'auteure souligne que l'approche discursive nous permet de voir l'Histoire des Idées Linguistiques « comme une histoire sujette à l'équivoque, aux relectures, aux divisions, aux différentes inscriptions théoriques, bref, à la critique des théories »(ORLANDI, 2002, p. 9, nous traduisons).

L'Histoire des Idées Linguistiques cherche à comprendre le fonctionnement du savoir linguistique dans différentes sociétés et époques. Elle n'est pas fermée sur la Linguistique Moderne, mais ouverte aux différents types de savoirs produits sur le langage humain. Les travaux en Histoire des Idées Linguistiques analysent la production de savoirs linguistiques depuis l'Antiquité. Selon Auroux (1992, p. 8-9), il y a eu trois révolutions technologiques depuis l'Antiquité jusqu'à nos jours qui ont contribué au développement des sciences du langage : (i) l'apparition de l'écriture (il y a 3000 ans) : «l'écriture est un des facteurs nécessaires pour l'apparition des sciences du langage » (AUROUX, 1992, p. 8); (ii) la grammatisation des langues du monde à partir de la Renaissance : «la Renaissance européenne est le point d'inflexion d'un processus qui conduit à la production de dictionnaires et de grammaires de toutes les langues du monde (et non seulement des langues vernaculaires européennes) » (AUROUX, 1992, p. 8) et (iii) l'informatisation des langues, dont les effets sont encore incertains. 
Dans ce travail, notre but est d'analyser la production de manuels de langage non sexiste et la manière dont ces publications formulent un savoir linguistique sur le sexisme. Nous considérons le manuel un outil technologique et un produit socioculturel de vulgarisation d'un savoir sur la langue. Basés sur des lois, des décrets etc., ils essaient de répondre aux discours féministes qui revendiquent une position légitimée pour la femme. Le but de ces manuels, où l'on peut remarquer la diffusion et le fonctionnement de ces savoirs dans la société, est de promouvoir une culture non sexiste où l'équité de genre soit marquée dans les usages linguistiques (GARCIA; SOUSA, 2016).

Conçus comme un outil linguistique ${ }^{1}$, les manuels font partie du processus de grammatisation ${ }^{2}$ d'une langue, puisqu'ils la décrivent et l'outillent. Ces outils mettent en évidence le sexisme linguistique et contredisent la norme linguistique courante. En outre, nous concevons les manuels comme un « objet socioculturel clairement identifiable par rapprochement/différenciation (d')avec d'autres produits socioculturels du monde savant » (PUECH, 1998, p. 15-16). Les manuels sont différents, par exemple, d'un dictionnaire. Inscrits dans un discours institutionnel, ils prescrivent et proscrivent certains usages : «à partir des règles grammaticales, les manuels présentent le fonctionnement de la langue et mettent en circulation un certain savoir sur cette langue-là dans la société » (GARCIA, 2017, p. 90, nous traduisons). D’après Puech (1998, p. 15-16), la manuélisation est un processus «par lequel les savoirs linguistiques s'exposent et se diffusent à des fins opératoires de transmission, appropriation, réinvestissement, plutôt que du produit à proprement parler ». Comme l'on verra, les manuels que nous analysons mettent en évidence un savoir linguistique sur le genre morphologique (masculin et féminin), mettent en question l'usage générique du genre masculin et montrent que ce geste empêche la visibilité linguistique de la femme. Pour promouvoir l'équité de genre et supprimer le sexisme dans le langage, ils proposent que le genre féminin soit marqué dans les usages linguistiques.

Ensuite, nous analysons le sexisme dans la langue et nous exposons son fonctionnement dans les usages linguistiques faits par les sujets lors de leur énonciation.

\section{LE SEXISME DANS LE LANGAGE}

Mills (2008, p. 1) explique que le langage est un espace de lutte entre les sexistes et les militantes pour les droits des femmes, une lutte qui porte sur le droit d'occuper certaines positions, de parler d'une certaine manière et d'occuper certains postes de

\footnotetext{
${ }^{1}$ «La grammaire n'est pas une simple description du langage naturel. Il faut la concevoir aussi comme un outil linguistique: de même qu'un marteau prolonge le geste de la main de l'homme et le transforme, une grammaire prolonge la parole naturelle, et donne accès à un corps de règles et de formes qui ne figurent souvent pas ensemble dans la compétence d'un même locuteur. Ceci est encore plus vrai dans le cas des dictionnaires : quelle que soit ma compétence linguistique, je ne maitrise certainement pas toute la grande quantité de mots qui figurent dans les grands dictionnaires monolingues » (AUROUX, 1992, p. 69).

2 «Par grammatisation, on doit entendre le processus qui conduit à décrire et à outiller une langue sur la base des deux technologies qui sont encore aujourd'hui les piliers de notre savoir métalinguistique : la grammaire et le dictionnaire » (AUROUX, 1992, p. 65). À notre avis, cette réflexion s'applique aussi aux manuels.
} 
travail. Selon l'auteure (MILLS, 2008, p. 10), le langage sexiste est un terme utilisé pour désigner toute une gamme de différents éléments. Par exemple, les usages de pronoms (le pronom «ils» pour désigner génériquement les deux sexes), les gros mots employés différemment par les hommes et par les femmes, les désignations du corps masculin et féminin etc. En outre, le terme sexisme fait référence, d'après Mills (2008, p. 10), à un ensemble de points de vue stéréotypés sur les femmes qui ne sont pas directement liés à un groupe d'usages linguistiques spécifique.

Selon Mills (2008), le sexisme fonctionne sur deux niveaux: un niveau institutionnel - représentatif des normes de la société - et un niveau individuel spécifique et lié aux normes sociales. L'auteure propose aussi une distinction entre le sexisme direct et le sexisme indirect (MILLS, 2008, p. 11-12). Le sexisme direct est l'emploi d'expressions offensantes contre la femme qui renforcent sa position délégitimée dans la société. C'est le sexisme le plus fréquemment mis en question par les mouvements féministes. Comme «réaction » aux critiques féministes, on assiste à l'apparition du sexisme indirecte, ironique et plein de «bonne humeur », où l'énonciateur n'assume pas directement son sexisme.

Cameron (1990, p. 14) affirme que, vis-à-vis la nécessité de proposer un langage non sexiste, le langage sexiste ne doit pas être vu comme la «nomination » du monde à partir d'une perspective masculiniste, mais plutôt comme un phénomène à plusieurs facettes, présent partout. D'après l'auteure (CAMERON, 1992), voici quelques lieux de manifestation du sexisme dans le langage quotidien :

1. dans certains termes d'affection qui sont en réalité des petites insultes ;

2. dans certaines phrases de drague qui explicitent la violence verbale contre les femmes ;

3. dans certaines insultes qui veulent exercer un contrôle social ;

4. dans la langue institutionalisée des dictionnaires et des grammaires.

Les petites insultes ${ }^{3}$ font référence aux termes d'affection appliqués aux femmes (« chérie », « ma puce », « ange », « bébé », « princesse ») qui marquent généralement une relation de supériorité entre l'homme et la femme.

En ce qui concerne les phrases de drague - d'après Cameron (1992), une forme de violence verbale contre les femmes -, elles peuvent être entendues comme la tentative masculine de contrôler l'espace public et la circulation des femmes. L'auteure souligne que ce genre de terme, de phrase etc. est plus employé par les hommes, ce qui explique sans doute une quantité supérieure d'insultes contre les femmes, surtout au niveau sexuel (CAMERON, 1991, p. 107). Les insultes contre les hommes font référence le plus souvent à l'homosexualité, en raison non seulement de l'homophobie, mais aussi du sexisme, puisque l'image de l'homme homosexuel est attachée à l'image féminine (CAMERON, 1992, p. 108).

Quant au contrôle social, les insultes contre les femmes construisent une fausse responsabilité de la part des femmes (leur comportement, leurs vêtements etc.) par rapport aux violences physiques et verbales qu'elles subissent. « La prolifération d'insultes

\footnotetext{
${ }^{3}$ On reprend ici une discussion menée par Loureiro (2014) sur les différents types de sexisme linguistique.
} 
sexuelles contre la réputation des femmes a le but de les contrôler » (LOUREIRO, 2014, p. 67). Autrement dit, ces termes veulent garder les femmes à la maison.

Finalement, les dictionnaires et les grammaires explicitent le sexisme présent dans les normes institutionalisées par les outils linguistiques.

Pour rendre possible l'usage non sexiste du langage, Cameron (1998) affirme qu'il faut que ces usages soient «acceptés » par les « gardiens du langage » (lexicographes, journalistes, éditeurs etc.). Pour être efficace, cette « réforme » doit être acceptée et même divulguée par ceux qui occupent les "positions d'influence ». D'une manière générale, les termes alternatifs suggérés par les féministes sont acceptés par ces «gatekeepers », car les maisons d'édition, les syndicats, les universités etc. adoptent de plus en plus des politiques sur le langage (non) sexiste. Toutefois, d'autres institutions, comme les «journaux de droite», ridiculisent les propositions féministes, vues comme du «politiquement correct». Il y a aussi ceux qui marquent leurs positions en employant expressément des termes « politiquement incorrects ».

Comme l'on verra, la féminisation de la langue est l'une des manières de combattre le sexisme.

\section{LA FEMINISATION DE LA LANGUE}

Le débat sur la féminisation de la langue n'est pas du tout homogène. Composé de toute une pluralité de positions, il montre que l'acte de désigner dépend du statut et de la visibilité des sujets dans la société. D’après Elmiger (2011, p. 72-73), féminiser la langue constitue une tentative de favoriser l'égalité entre les sexes, à partir de l'idée que l'existence et l'utilisation des désignations féminines produisent l'égalité (de rémunération, de droits, d'opportunités etc.) dans la « réalité sociale ».

Iváñez (1996, p. 71) souligne que la féminisation de titres, fonctions et métiers illustre quelques exemples de changement linguistique. Le point de départ de ces changements est une réalité de nature sociologique : l'accès des femmes au monde du travail, majoritairement occupé par des hommes. On assiste non seulement à l'invention de nouvelles désignations pour ces femmes qui occupent des postes traditionnellement masculins, mais aussi aux réactions qui conditionnent l'adoption (ou le refus) systématique de la féminisation linguistique. Autrement dit, Iváñez explique que, d'une manière générale, la féminisation de la langue peut être associée à l'idéologie féministe, qui essaie de changer certains usages linguistiques afin d'attribuer à la femme un statut plus adéquat. Par exemple, l'emploi générique du masculin dans le cas des titres, des fonctions et des métiers serait un reflet du sexisme dans la langue et cacherait linguistiquement la participation de la femme dans la société.

Pour Iváñez (1996), l'état actuel de cette question ne révèle pas forcément l'absence d'un consensus sur la nécessité de féminiser ou non les titres, mais plutôt le fonctionnement de deux problèmes spécifiques :

1. D'une part, il y a l'hésitation entre plusieurs formes possibles (une chercheur, une chercheure, une chercheuse) ou entre deux formes qui n'ont pas exactement le même sens (une docteure, une doctoresse) ; 
2. D'autre part, malgré la généralisation du féminin, on peut constater que l'usage du masculin (terme non marqué d'opposition des genres) est lié à cette même hésitation et se présente dans les cas où le mot en question exprime le métier ou le titre de l'individu. L'emploi du masculin n'est pas du tout fréquent si le mot exprime l'individu lui-même (IVÁÑ̃EZ, 1996, p. 72).

Par rapport au premier problème, il faut prendre en compte l'influence de la planification linguistique et l'intervention de l'État pour l'adoption décisive de certains usages linguistiques non sexistes. Par rapport au deuxième problème, Iváñez (1996) croit que les genres seront préservés et que, d'un point de vue structurel, les deux formes d'exprimer le métier ou le titre continueront à exister : le masculin générique et le féminin spécifique. Comme l'affirme Iváñez (1996, p. 72), il faudrait une vraie prise de position idéologique pour imposer la forme féminine dans le domaine des métiers et des fonctions exécutés par les femmes.

Abbou (2011, p. 279) affirme qu'il y aurait trois raisons pour la féminisation de la langue : la visibilité (de la femme dans les usages linguistiques), la démasculinisation des formes génériques, et la langue comme un lieu de lutte politique. Pour l'auteure (2011, p. 301), la féminisation est donc une lutte et un outil politiques.

C'est la démasculinisation de la langue, articulée à sa féminisation, que l'on peut remarquer dans le manuel que nous analysons ci-dessous.

\section{LES MANUELS ET LA LANGUE: LE MASCULIN ET LE FEMININ EN OPPOSITION}

Dans notre recherche sur la féminisation de la langue, nous avons trouvé les manuels suivants, qui proposent un usage non sexiste du langage ${ }^{4}$ :

- Guía del lenguaje para el ámbito de la empresa y el empleo (Eulàlia Lledó Cunill, 2011).

- Guía del lenguaje para el ámbito de la cultura (Eulàlia Lledó Cunill, 2010).

- Guía del lenguaje para el ámbito del deporte (Eulàlia Lledó Cunill, 2009).

- Guía del lenguaje para el ámbito de la salud (Eulàlia Lledó Cunill, 2009).

- Guía del lenguaje para el ámbito educativo (Eulàlia Lledó Cunill, 2008).

- Las profesiones de la A a la Z (Eulàlia Lledó Cunill, 2006).

- Hablamos de leyes (Charo Guerrero Martin, Eulàlia Lledó Cunill, 2008).

- Nombran: la representación del femenino y el masculino en el lenguaje (Eulàlia Lledó Cunill, 1995).

- Langage et sexisme (Préfecture de la Réunion, s.d.).

- L'égalité s'écrit: guide de rédaction épicène (Bureau de l'égalité entre les femmes et le hommes, 2007).

- Petit guide pour un langage non sexiste (Céline Orban, 2012).

\footnotetext{
${ }^{4}$ Cette recherche a été faite sur Google.com. On a tapé les termes (en portugais, espagnol et français) langage sexiste, féminisation de la langue, manuels, masculin comme terme générique. Tous ces outils linguistiques sont disponibles en ligne. On a pas pris en considération les manuels qui ne peuvent pas être consultés sur Internet.
} 
- Femme, j'écris ton nom... Guide d'aide à la féminisation des noms de métiers, titres, grades et fonctions (Centre National de la Recherche Scientifique, Institut National de la Langue Française, 1999).

- Manual para o uso não sexista da linguagem: o que bem se diz, bem se entende (Secretaria de Políticas Públicas para as Mulheres do Governo do Estado do Rio Grande do Sul, 2014).

L'usage non sexiste du langage, conséquence de la féminisation de la langue, fait partie d'un débat aussi politique que linguistique, dont l'origine se trouve surtout dans les mouvements féministes et de lutte pour l'égalité des genres. Selon Zoppi-Fontana (2015, p. 226-227), la féminisation du langage

\begin{abstract}
[dénonce] les effets sociaux et idéologiques de l'emploi de formes linguistiques (lexicales et morphologiques) qui reproduisent et renforcent des représentations négatives des relations de genre dans la société. En général, on pense que les formes lexicales négatives ne se réfèrent qu'à l'identité de genre (par exemple, bicha, traveco, sapatão, cadela etc.), mais on peut remarquer aussi des formes de nature grammaticale, qui font partie de la structure même du système linguistique, comme l'accord de genre et plus spécifiquement l'emploi du masculin comme genre générique non marqué qui désigne les deux genres grammaticaux. Dans le contexte de ce débat, on peut penser aussi aux postes et aux fonctions traditionnellement masculins (par exemple, la polémique qui a eu lieu récemment au Brésil sur l'emploi du terme presidenta).
\end{abstract}

D’une manière générale, les manuels cherchent à rendre visibles quelques fonctions et métiers exécutés par les femmes et qui traditionnellement n'ont que la forme générique masculine. En didactisant et mettant en circulation un autre dire sur le genre morphologique dans le processus de manuélisation, «les manuels mettent en relief les préjugés de genre matérialisés dans la société. Ces outils produisent un savoir linguistique qui marque l'absence d'égalité (non seulement linguistique) entre la femme et l'homme dans une société qui fait un certain usage de sa propre langue »(GARCIA, à paraître).

Comme l'on a déjà expliqué, la proposition de féminisation des langues est liée à certaines actions politiques, juridiques et linguistiques et à la critique féministe développée à partir des années 1970. Selon Elmiger (2011, p. 72), le cas de la féminisation (de textes officiels) illustre les efforts réalisés par une communauté afin de modifier l'utilisation de sa langue et, par conséquent, de valoriser le statut des femmes dans sa société.

Il y a de nombreux pays et régions francophones qui ont développé des moyens pour «encourager » l'usage de la langue féminisée. Le Québec ${ }^{5}$, par exemple, est la première région francophone à prendre des décisions politiques sur la féminisation de la langue. Les premières recommandations lexicales datent des années 1970: en 1976, un ministre canadien consulte L'Office de la Langue Française afin de décider sur la possibilité de féminiser les désignations de métiers ou sinon d'adopter les formes épicènes, comme en anglais. En 1979, dans le rapport Pour les Québécoises : égalité et indépendance du Conseil du Statut de la Femme, L'Office de la Langue Française se déclare pour «l'utilisation de formes féminines dans tous les cas possibles» (BOUCHARD et al., 1999, p. 8, apud ELMIGER, 2011, p. 73). Pendant les années 1980,

${ }^{5}$ Pour d'autres exemples, voir Elmiger (2011, p. 73-76). 
on assiste à la sortie d'autres publications, comme Titres et fonctions au féminin : essai d'orientation de l'usage (Office de la Langue Française, 1986). Selon Elmiger (2011, p. 73-74), les premiers textes portent fondamentalement sur la formation des noms féminins (l'aspect lexical de la féminisation). Pourtant, l'idée de féminiser tous les noms communs de personnes en fonction du contexte ( «féminisation du discours ») est plus récente: le guide Pour un genre à part entière (DUMAIS, 1988) expose plusieurs questions concernant la féminisation de textes et préconise l'utilisation du système de deux formes, féminine et masculine. Des institutions publiques et privées québécoises publient aussi d'autres guides, comme Au féminin, élaboré par l'Office de la Langue Française (1991) et $\grave{A}$ Juste Titre (1994).

En France, comme l'explique Elmiger (2011), la question (uniquement lexicale) des désignations féminines est d'abord discutée par une commission de terminologie. La commission, en ayant le but de féminiser les noms des métiers et des travaux, est présidée par Benoîte Groult. Depuis sa création, en 1984, la «Commission Groult » a subi des attaques violentes venues du public et de l'Académie Française. Les résultats des travaux de la commission ont été publiés sous le titre Circulaire du 11 Mars 1986 relative à la féminisation des noms de métier, fonction, grade ou titre. D’après Elmiger (2011, p. 7475), c'était un moment crucial de la vie politique française: la publication sort quelques jours avant l'arrivée de Jacques Chirac au poste de Premier Ministre. Pour Elmiger (2011, p. 74-75), le nouveau gouvernement (« de droite »), successeur d'un gouvernement « de gauche », ne «se sent pas obligé » d'adopter les recommandations dictées par la circulaire. Dix ans plus tard, également dans le contexte d'un changement de gouvernement, la question de la féminisation est reprise dans une nouvelle circulaire (Circulaire du 6 Mars 1998 relative à la féminisation des noms de métier, fonction, grade ou titre). Le premier ministre Lionel Jospin réagit à la décision de son équipe, composée de quatre femmes qui revendiquaient leur droit de se faire appeler "Madame la Ministre ». Deux rapports, dirigés par le premier ministre lui-même, arrivent à des conclusions différentes. D'une part, comme l'explique Elmiger (2011, p. 75), la Commission Générale de Terminologie et Néologie ${ }^{6}$ - COGETER 1998 - approuve la féminisation des noms de métiers ou de travaux pour désigner les femmes individuellement, mais se prononce contre l'utilisation des noms féminisés dans les textes juridiques. Selon la commission, il faudrait distinguer entre la désignation d'un individu et l'utilisation de noms communs de personnes pour désigner un changement officiel. D'autre part, l'Institut National de la Langue Française (INaLF) publie un guide destiné au grand public (Femme, j'écris ton nom, 1999, analysé dans ce travail), composé de règles et de recommandations sur la féminisation générale du lexique. Comme la circulaire de 1986, Elmiger (2011, p. 75) affirme que ce guide, qui date de 1999, se limite à traiter des aspects lexicaux de la féminisation.

En Belgique francophone, la réflexion sur la féminisation de la langue arrive en 1993, à partir du Décret du 21 Juin 1993 relatif à la féminisation des noms de métier, fonction, grade ou titre, publié par le Conseil de la Communauté Française. Le décret oblige que les institutions subordonnées au conseil utilisent des termes féminins pour désigner les femmes, mais il ne se prononce pas sur les formes qui doivent être employées. Cette lacune est corrigée plus tard avec la publication d'un nouveau document de la Communauté Française, qui établit les règles de féminisation des noms de métier,

\footnotetext{
${ }^{6}$ Aujourd'hui la commission s'appelle La Commission d'enrichissement de la langue française.
} 
fonctions, grades et titres. Ces deux textes officiels produisent deux publications : un guide destiné au grand public, Mettre au féminin, qui explique les règles morphologiques de formation du féminin à partir de son correspondant masculin ; et les Recommandations Générales du Conseil Supérieur de Langue Française et Surtout une Liste de Noms de Métier, Fonction, Grade ou Titre. D'après Elmiger (2011, p. 75), les règles syntaxiques préconisées par les publications belges se limitent à recommander « l'accord au féminin » dans le cas des déterminants, des adjectifs et des participes. Quant à la féminisation du discours, les recommandations sont assez vagues : «il est recommandé de ne pas abuser de l'emploi générique des noms masculins, mais il ne propose pas de solutions viables » (ELMIGER, 2011, p. 75).

En Suisse, en raison de son fédéralisme, la question de la féminisation n'est pas homogène dans toutes les instances (fédérale, cantonale et municipale). La Chancellerie Fédérale et les Services Linguistiques s'occupent de l'utilisation de termes féminisés au niveau national. Un groupe de travail interdépartemental a publié en 1991 un rapport intitulé La Formulation non sexiste des textes législatifs et administratifs. Ce rapport, écrit en français, allemand et italien (les autorités cherchent à présenter des solutions applicables à toutes les langues du pays), ne porte pas sur la féminisation du lexique, mais plutôt sur la composition de textes, surtout juridiques et législatifs. La solution proposée, appelée de "créative », est la combinaison de différentes stratégies pour éviter l'emploi générique des formes masculines.

En 1993, le Conseil Fédéral décrète que le lexique et les documents normatifs doivent être féminisés. Par contre, le Conseil affirme que seulement « les textes législatifs en langue allemande seront rédigés conformément à la solution dite créative [...] [et que] la pratique actuelle sera maintenue en ce qui concerne les textes français et italiens » (apud ELMIGER, 2011, p. 76).

En Suisse, comme 1'explique Elmiger (2011, p. 76), la plupart des décisions relatives à la féminisation de la langue est prise par les autorités cantonales. Les régions francophones n'ont ni les mêmes critères ni les mêmes buts à l'égard de la féminisation linguistique: il y a ceux qui assument une posture active et développent une administration qui s'occupe de ce genre de discussion, et il y a aussi ceux qui n'adoptent aucune mesure particulière. Au niveau régional, il y a eu la publication du Dictionnaire féminin-masculin des métiers, des titres e des fonctions (1990, réédité en 1999) et du guide Écrire les genres : guide romand d'aide à la rédaction administrative et législative épicène (MOREAU, 2001).

À partir de ce panorama général, on passe à l'analyse du manuel Femme, j'écris ton nom...

Le manuel Femme, j'écris ton nom... Guide d'aide à la féminisation des noms de métiers, titres, grades et fonctions a été publié en 1999 par le Centre National de la Recherche Scientifique et l'Institut National de la Langue Française. Élaboré par AnneMarie Becquer, Nicole Cholewka, Martine Coutier, Marie-Josèphe Mathieu et Bernard Cerquiglini, le manuel présente la structure suivante: Préface (écrite par le premier ministre Lionel Jospin) ; Introduction; Aperçu historique: la féminisation au cours des siècles ; Règles de féminisation des noms de métiers, titres, grades et fonctions ; Objections et difficultés; Constitution et présentation de la liste ; Rôle de l'adjectif dans la terminologie des métiers ; En guise de conclusion ; Références bibliographiques et Guide d'aide à la féminisation des noms de métiers, titres, grades et fonctions. 
Dans cette analyse, nous nous penchons sur un extrait des règles de féminisation et sur la partie finale du manuel.

Dans la préface, le premier ministre Lionel Jospin, sympathisant de la proposition de féminisation des noms de métier, titres, grades et fonctions, rappelle que la France est un pays particulièrement intéressé aux questions linguistiques et affirme que le gouvernement ne doit pas imposer une norme, car «la liberté d'expression, une des libertés les plus fondamentales dans une démocratie, suppose le droit pour chacun d'utiliser la langue comme il l'entend » (BECQUER et al., 1999, p. 5). En même temps, le premier ministre affirme que le gouvernement doit donner l'exemple dans le domaine des services publics. À la demande du premier ministre, la Commission Générale de Terminologie et Néologie élabore un rapport sur la féminisation des noms et suggère quelques usages spécifiques de titres, métiers, grades et fonctions. Finalement, le premier ministre montre qu' « il n'y a pas de difficulté à féminiser la plupart des métiers, grades, titres et fonctions » (BECQUER et al., 1999, p. 6). La question de la féminisation est donc «symbolique et non linguistique » (BECQUER et al., 1999, p. 5).

En exposant les règles de formation des noms au féminin, le manuel réaffirme la position du premier ministre et montre que la structure de la langue permet la féminisation de la plupart des noms de métier, grades, fonctions et titres. Ce genre de formation a même été déjà fait au cours des siècles, comme l'on peut lire dans la section Aperçu historique : la féminisation au cours des siècles. L'emploi du féminin indique que le rôle joué par les femmes dans certains domaines du monde professionnel est symboliquement effacé à travers le langage.

Cet outil linguistique se limite à exposer les règles de formation du genre féminin en tant que catégorie morphologique binaire - masculin et féminin. Il ne propose pas, par exemple, une discussion sur la construction/identification socioculturelle de genre. Son but est de populariser le fonctionnement de la féminisation lexicale, ce qui justifie l'inclusion d'une liste à la fin du manuel où l'on présente un ensemble de vocables de métiers, titres, grades et fonctions au masculin et au féminin. Dans ce sens, féminiser la langue signifie mettre en évidence le genre féminin et «vulgariser», comme l'affirme Puech (1998), un savoir sur la langue, comme l'on peut voir ci-dessous :

Figure 1 - Femme, jécris ton nom...

\section{Le déterminant}

Dans tous les cas, la féminisation implique l'utilisation d'un déterminant féminin, pour les désignations simples comme pour les désignations complexes : la, une, cette... : la députée, une juge, cette agente de change, la fondée de pouvoir...

\section{Noms se terminant au masculin par une voyelle}

Il s'agit de termes non suffixés, comme apprenti, architecte, gendarme, juge, ministre, vétérinaire, ou suffixés/composés (-é, -logue, -iste, etc.).

Source: Becquer et al., 1999, p. 22. 


\section{Figure 2 - Femme, jécris ton nom...}

\section{2.a. Noms se terminant par -e}

8 - Voir supra, note 5 ,

La forme féminine est identique à la forme masculine (forme épicène) $)^{8}$,
ex. : une architecte, une astronaute, une cadre, une capitaine, une commissaire, une diplomate, la garde (des Sceaux), une garde-malade, une gendarme, une géologue, une interne, une interprète, une juge, une manœuvre, une ministre, une paysagiste, une peintre, une psy- chiatre, une vétérinaire..

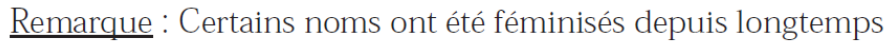
à l'aide du suffixe -esse : hôtesse, mairesse, maitresse, poétesse... Ce suffixe étant aujourd'hui senti comme désuet, voire dévalorisant, on a préféré ne plus y avoir recours. Seuls les emplois consacrés sont retenus (une hôtesse, une maitresse d'école) ; les emplois encore partiellement en usage sont toujours admis, à côté des formes épicènes proposées ou déjà concurrentes dans l'usage : une maire ou mairesse, une maitre ou maitresse (d'hôtel, de conférences, etc.), une poète ou poétesse...

Source : Becquer et al., 1999, p. 22.

Le savoir linguistique sur le genre est exposé et divulgué avec le but d'expliquer la formation du féminin des noms de métiers, fonctions, grades et titres. Le manuel présente une didactisation de ce savoir linguistique et aussi une critique à l'usage du masculin comme forme générique, qui serait une erreur et une agression contre la femme :

\section{Figure 3 - Femme, jécris ton nom...}

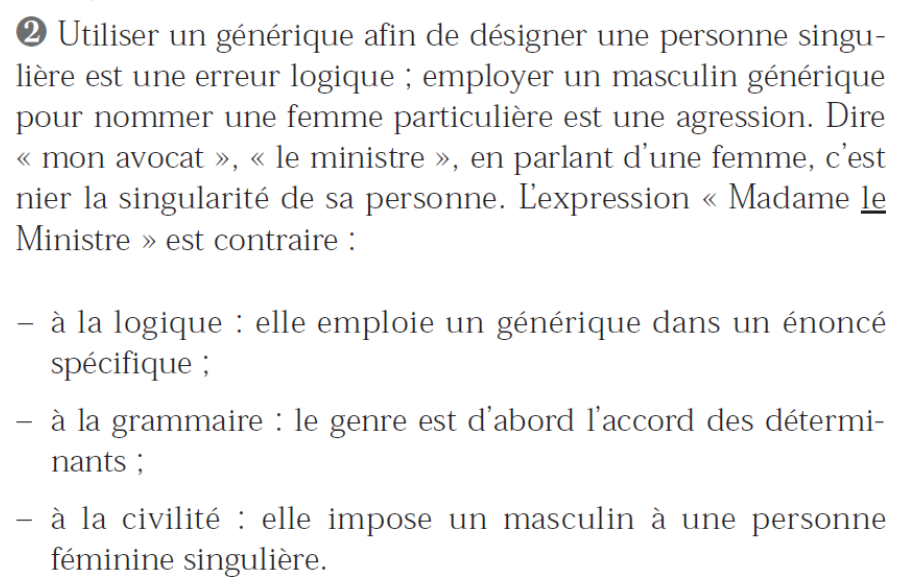

Source: Becquer et al., 1999, p. 39.

Le manuel propose l'association entre le genre et le sujet empirique. Par exemple : s'il s'agit d'une femme, on emploie le genre féminin. Le masculin en tant que forme générique dans les usages linguistiques rend invisibles la femme et le métier qu'elle exerce.

À la fin du manuel, il y a une liste de noms de métiers, titres, grades et fonctions féminisés. Cette liste a été élaborée à partir de guides et dictionnaires de plusieurs nations francophones (Belgique, Québec, Suisse) et du Répertoire Opérationnel des Métiers et 
des Emplois (ROME), qui présente plus de 10000 dénominations actuelles. Il y a des métiers libéraux, militaires, médicaux, religieux, du droit, des arts, des sports etc., organisés par ordre alphabétique, accompagnés de sa forme masculine et de sa règle de formation.

Figure 4 - Femme, jécris ton nom...

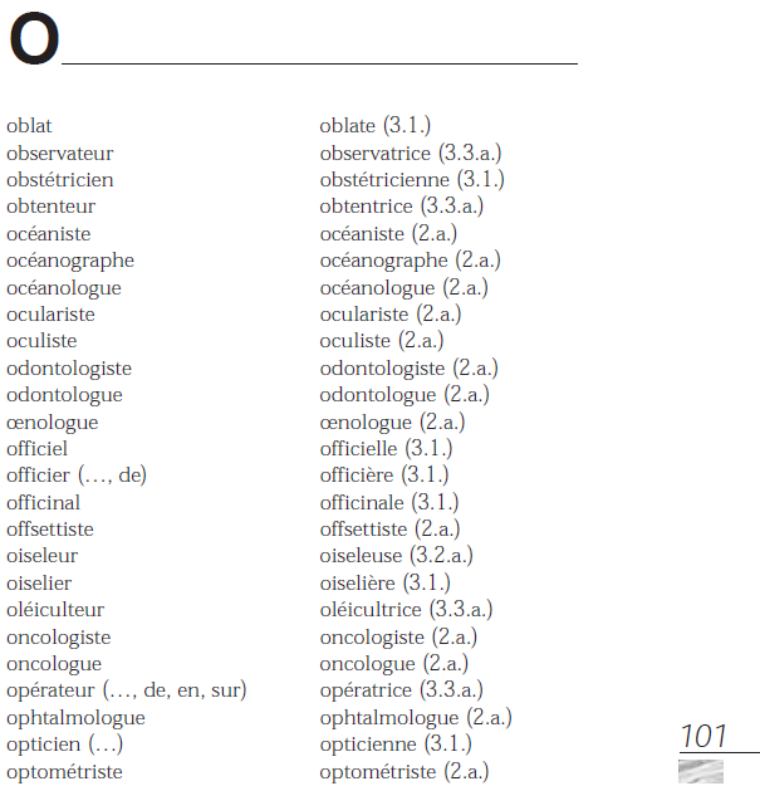

Source : Becquer et al., 1999, p. 101.

Le manuel produit un savoir linguistique sur la formation du genre féminin en français. L'emploi du masculin dans le cas de métiers, fonctions, titres et grades occupés par des femmes marque un fonctionnement symbolique dans la société qui annule linguistiquement la femme et son métier. Le manuel et la liste de désignations professionnelles réaffirment l'existence des femmes, malgré le sexisme dans la société et (par conséquent) dans la langue.

6 POUR CONCLURE...

Dans ce travail, on a cherché à réfléchir sur le processus de manuélisation d'un langage non sexiste - dans notre cas spécifique, la féminisation des noms. En reprenant les questions posées dans l'introduction, nous pouvons affirmer après notre analyse que la manuélisation d'une langue féminisée produit un savoir sur le genre morphologique, ce qui permet d'expliciter la formation de la forme féminine des noms de métiers, fonctions, grades et titres. Pourtant, bien plus qu'expliquer les règles de formation du féminin, le manuel Femme, j'écris ton nom ... met en évidence que les femmes peuvent exercer (ou exercent déjà) n'importe quel métier et que l'emploi du masculin comme forme générique est une erreur et une agression contre la femme.

Ainsi, la manuélisation d'un langage non sexiste est un acte politique de redonner à la femme, à travers la langue, sa vraie place dans la société. 
ABBOU, J. L'antisexisme linguistique dans le brochures libertaires: pratiques d'écriture et métadiscours. Université de Provence. Aix-Marseille I, 2011. Disponible sur <https://tel.archives.ouvertes.fr/tel00643802>. Dernier accès, février 2015.

AUROUX, S. A revolução tecnológica da gramatização. Campinas : Editora da Unicamp, 1992. BECQUER, A-M.; CHOLEWKA, N; COUTIER, M; MATHIEU, M-J; CERQUIGLINI, B. Femme, j'écris ton nom. Guide d'aide à la féminisation des nomns de métiers, titres, grades et fonctions. Paris : Centre National de la Recherche Scientifique, Institut National de la Langure Française, 1999.

CAMERON, D. A Feminist Critique of Language. London : Routledge, 1990. Feminism and Linguistic Theory. $2^{\mathrm{e}}$ édition. New York: Palgrave, 1992.

The Feminist Critique of Language: A Reader. London: Routledge, 1998.

DUMAIS, H. Pour un genre à part entière: guide pour la rédaction de textes non sexistes. Québec: Publications du Québec, 1988.

GARCIA, D.A.; SOUSA, L.M.A. e. A manualização do saber linguístico e a constituição de uma linguagem não sexista. Revista Línguas \& Letras. Cascavel, v. 17, n. 35, p. 86-106, 2016.

GARCIA, D. A. Manualização de uma linguagem não sexista: uma política de equidade de gênero? In: SOARES, A. S. F.; GARCIA, D.A. Inquietações de gêneros e sexualidades: leituras na contemporaneidade. Porto Alegre: Evangraf, 2017.

ELMIGER, D. Féminisation de la langue française: une brève histoire des positions politiques et du positionnement linguistique. In: DUCHÊNE, A.; MOÏSE, C. Langage, genre et sexualité. Québec : Éditions Nota Bene, 2011.

IVÁÑEZ, M. P. L'influence de la planification linguistique dans la féminisation des titres en France et au Québec : deux résultats différents en ce qui a trait à l'usage. Revue Québécoise de Linguistique, v. 24, n. 2, p. 71-106, 1996.

LOUREIRO, M. O gênero no discurso de opinião na Imprensa Portuguesa. Covilhã: Livros Labcom, 2014. Disponible sur <www.livroslabcom.ubi.pt/pdfs/20140710_201401_mloureiro_generodiscursoopiniao.pdf >. Dernier accès, février 2015.

MILLS, S. Language and sexism. New York: Cambridge University Press, 2008. Disponible sur <www.english.fullertion.edu/publications/cln/clnarchives/2009spring/langsex.pdf>. Dernier accès, février 2015.

MOREAU, T. Écrire les genres: guide romand d'aide à la rédaction administrative et législative épicène. Gèneve: État de Gèneve, 2001.

ORLANDI, E.P. (Org.). História das idéias lingüísticas: construção do saber metalingüístico e constituição da língua nacional. Campinas: Pontes; Cáceres: Unemat, 2001.

Lingua e conhecimento lingüístico. Campinas : Pontes, 2002.

PUECH, C. Manuélisation et disciplinarisation des savoirs de la langue. L'énonciation. Les Carnets du Cediscor, 1998. Disponible sur <http://cediscor.revues.org/267> . Dernier accès, février 2015.

ZOPPI-FONTANA, M. G. Língua oficial e políticas públicas de equidade de gênero. Línguas $e$ Instrumentos Linguísticos. Campinas, n. 36, p. 221-243, jul.-dez. 2015.

Recebido em: 12/08/17. Aprovado em: 16/07/18.

Title: Sexism and feminization of language: a process of manualization Author: Dantielli Assumpção Garcia

Abstract: Based on theoretical perspective of Discourse Analysis in conjunction with the History of Linguistic Ideas, this paper analyzes the manual Femme, j'écris ton nom... Guide d'aide à la féminisation des noms de métiers, titres, grades et fonctions (1999), aiming to reflect on what is the process of manualization of a non-sexist language, in which the feminization of language is present, and how it is established. By a feminized language manualization, it produces a knowledge about the morphological gender, explaining how to form the feminine of the names of professions, functions, degrees, and titles, and the Manual 
Femme, jécris ton nom marks that women can and carry on the different professions listed there in their guide, and that the male form as generic is a mistake and an aggression to the woman.

Keywords: Feminization. Manualization. Discourse Analysis. History of Linguistic Ideas.

Título: Sexismo y feminización del lenguaje: un proceso de manualización

Autora: Dantielli Assumpção Garcia

Resumen: Desde la articulación teórica entre el Análisis del Discurso y la Historia de las Ideas Lingüísticas, este trabajo analiza el manual Femme, j'écris ton nom... Guide d'aide à la féminisation des noms de métiers, titres, grades et fonctions (1999), con el objetivo de reflexionar sobre la constitución del proceso de manualización de un lenguaje no sexista, marcado por la feminización de la lengua. La manualización de una lengua feminizada produce un saber sobre el género morfológico, lo que permite explicitar la formación de la forma femenina de los nombres de profesiones, funciones, grados y títulos, y el manual Femme, j'écris ton nom... pone en evidencia que las mujeres pueden ejercer (o ya ejercen) cualquier profesión, y que el empleo del masculino como forma genérica es un error y una agresión contra la mujer.

Palabras-clave: Feminización. Manualización. Análisis del Discurso. Historia de las Ideas Lingüísticas.

Título: Sexismo e feminização da língua: um processo de manualização

Autora: Dantielli Assumpção Garcia

Resumo: Partindo da articulação teórica entre a Análise de Discurso e a História das Ideias Linguísticas, este trabalho analisa o manual Femme, j'écris ton nom... Guide d'aide à la féminisation des noms de métiers, titres, grades et fonctions (1999), com o objetivo de refletir sobre a constituição do processo de manualização de uma linguagem não sexista, marcada pela feminização da língua. A manualização de uma língua feminizada produz um saber sobre o gênero morfológico, o que permite explicitar a formação da forma feminina dos nomes de profissões, funções, graus e títulos, e o manual Femme, j'écris ton nom... põe em evidência que as mulheres podem exercer (ou já exercem) qualquer profissão, e que o emprego do masculino como forma genérica é um erro e uma agressão contra a mulher.

Palavras-chave: Feminização. Manualização. Análise de Discurso. História das Ideias Linguísticas.

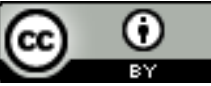

Este texto está licenciado com uma Licença Creative Commons Atribuição 4.0 Internacional. 